\title{
Prediction of Two-Phase Flow Patterns Using Machine Learning Algorithms
}

\author{
Naief Almalki ${ }^{1}$, Wael H. Ahmed ${ }^{1}$ \\ ${ }^{1}$ School of Engineering, University of Guelph \\ Guelph, ON, Canada, N1G 2W1 \\ nalmalki@uoguelph.ca, ahmedw@uoguelph.ca
}

\begin{abstract}
Predicting the two-phase flow pattern prediction is important to many industries such as power generation and oil and gas; for example, knowing the type of flow pattern is crucial for an accurate calculation of the pressure on the system. The transient nature of two-phase flows makes analyzing and predicting the flow pattern for a normal straight pipe a very complex procedure. The situation becomes more complex when a piping component is disturbing the fully developed flow in a straight pipe. In this work, the flow pattern downstream of an orifice was experimentally investigated for an intermittent flow pattern at orifice-to pipe area ratios of 0.14 , 0.25 and 0.56. The flow pattern downstream of the orifice was identified using a probability density function (PDF) of the time signal void fraction as well as identified using a high-speed imaging system. All tests were presented by the calculated superficial velocity of the mixture based on the area of the orifice being used and the volumetric quality. The predicted flow pattern was identified using a Machine Learning Algorithm known as the Classification Learner environment in MATLAB. This method was able to predict the flow pattern downstream of the orifice with a total error of $\pm 9 \%$.
\end{abstract}

Keywords: Two-phase flow, Flow pattern, Orifice, Machine learning.

\section{Introduction}

A multiphase flow, in which two or more phases flow together in a channel, is usually categorized based on the configuration of the phases or the so-called flow pattern. Similar to a single-phase flow, which is classified as laminar or turbulent, multiphase flow is classified based on the flow pattern in either a horizontal or a vertical channel; however, even in simple cases of two-phase flow involving one component, such as steam-water, or two components, such as air-water or oil-gas, the definition of the flow pattern is more complex. Although the types of flow patterns in horizontal or vertical flows are well-defined, the transition mechanisms are not fully understood. The situation becomes more complex when a piping component is present in the pipeline, as this introduces a momentum change in the phases. Despite this fact, the flow pattern downstream of a piping component has been studied less often than that for a straight pipe.

The flow pattern in a straight pipe has been investigated for different types of orientations. In all cases, the type of flow pattern was identified either through visual observations or by using pressure or void fraction signals. Jones and Zuber [1] showed that the probability density function of the void fraction signal has a unique shape for each flow pattern. Three PDF shapes were defined for bubbly, slug, and annular flows. A bubbly flow has one PDF peak with an average value close to zero. In contrast, an annular flow has one PDF peak with an average void fraction close to one. Slug flow has two peaks, and combines both the bubbly and the annular flow types. Mandhane et al. [2] used a total of 5935 experimental observations for different fluid properties to establish a convenient flow pattern map for a horizontal pipe based on gas and liquid superficial velocities. Taitel and Dukler [3] established a mechanistic flow pattern map that can be used at different inclinations, flow properties, and dimensions.

With the advancements in computer analysis and artificial intelligence, new methods have been implemented in order to model the flow patterns in two-phase flow. Mi et al. [4] used a neural network method to model the flow pattern in a vertical pipe. They simulated the impedance signal for annular, churn, slug, and bubbly flow patterns and input the results into a supervised neural network model.

Trafalis et al. [5] implemented a multiclass support vector machine learning algorithm (MSVM) to model flow patterns in vertical and horizontal pipes. This model was built based on gas and liquid superficial velocities and the pipe diameter to predict the flow pattern transitions. Their results showed that MSVM was more effective than theoretical correlations in predicting flow pattern transitions. 
Wang and Zhang [6] used electrical capacitance tomography (ECT) data to model the flow pattern of oil-gas twophase flow based on a support vector machine (SVM) algorithm. The ECT has a dimension of 66 that was reduced to 14 using principal component analysis. Their results were highly accurate regarding flow pattern classification. They recommend that the SVM method be implemented on a small dataset.

Shaban et al. [7] used differential pressure signals as the input to create a flow pattern map using an elastic mapping technique. Different flow patterns were investigated for air-water two-phase flow in a vertical pipe. The elastic mapping method is a machine learning tool that is used to reduce the number of dimensions of data to be presented in a twodimensional map. The map that was created showed well-defined and clustered data of the same types of flow patterns.

Al-Naser et al. [8] implemented MATLAB and its built-in neural network algorithm to detect the flow pattern in a horizontal pipe. The authors used the unified model simulator to generate the input for different flow patterns. They created a general 3-D flow pattern map based on the liquid Reynolds number, the gas Reynolds number, and the pressure drop multiplier for different dimensions and fluid properties. They showed that based on the natural logarithm of the three dimensions, the model can accurately predict up to $97 \%$ of the flow patterns.

Ezzatabadipour et al. [9] used deep machine learning to predict annular, bubble, dispersed bubble, intermittent, stratified smooth, and stratified wavy flows at different pipe inclinations for pipes that were 1 or 2 inches in diameter. They used a feedforward neural network known as a multilayer perceptron (MLP) to train $60 \%$ of a total of 5676 data points with $20 \%$ for validation and $20 \%$ for testing. The results showed that the method was able to accurately predict the flow pattern.

Research on two-phase flow through orifices has focused mainly on the local pressure drop. Flow patterns downstream of an orifice have received relatively little attention regardless of the importance of this topic. The momentum of the phases increases dramatically as the mixture passes through the orifice. Ahmed [10] showed that piping degradation and failure were very common downstream of the orifice. Ahmed et al. [11] also showed that flow accelerated corrosion was greatest at a distance of 5D downstream of the orifice for both single- and two-phase flows. Bamidele et al. [12] showed that the flow pattern downstream of an orifice directly affected the vibration amplitude and direction.

The aim of this paper is to investigate the flow pattern downstream of an orifice in a horizontal pipe. To the best of the authors' knowledge, the prediction of the flow pattern in such a case has not been previously investigated. The initial flow pattern in the fully developed region upstream of the orifice was an intermittent flow pattern. Area ratios of 0.14 , 0.25 , and 0.56 were considered, as well as a that of a straight pipe without an orifice. The flow pattern was identified using a void fraction sensor installed within 5D downstream of the orifice as well as by using a high-speed camera. The identified flow patterns were used as input to model the flow pattern downstream of the orifice using machine learning algorithms in MATLAB.

\section{Experimental Facility}

The experimental facility was constructed to simulate air-water flows in a closed loop at the ambient temperature, as shown in

Fig. . Deionized water was pumped from a 310-L tank by a centrifugal pump controlled by a variable frequency drive. An Omega turbine flow meter with a reading accuracy of $\pm 1.5 \%$ was used to measure the rate of the liquid flow. Air was supplied from the main laboratory supply line, controlled by four rotameters, and measured by an Omega FMA6718 gas flow meter with an accuracy of $\pm 1 \%$ full-scale and a total range of $100 \mathrm{~L} / \mathrm{min}$. A two-phase annular mixer was used. This mixer was made of a stainless-steel pipe that was $80 \mathrm{~mm}$ long and $25.4 \mathrm{~mm}$ in diameter. Air was injected into the stream of water through a total of 64 holes that were $1 \mathrm{~mm}$ in diameter. These holes were distributed equally into eight sets spaced at $10 \mathrm{~mm}$ apart and machined onto the stainless-steel pipe. Two straight, clear polycarbonate pipes that were $2.4 \mathrm{~m}$ long were installed upstream and downstream of the orifice to allow for a visual inspection of the flow pattern. The pressure was measured at nine and thirteen locations upstream and downstream of the orifice, respectively, using pressure taps made of clear tubing to ensure that no air was trapped inside. This avoided inaccurate measurements. These pressure taps were connected to a mechanical pressure scanner from Scannivalve $\bigcirc$. The output of the pressure signal from the pressure scanner was then connected to two DP15 differential pressure sensors 


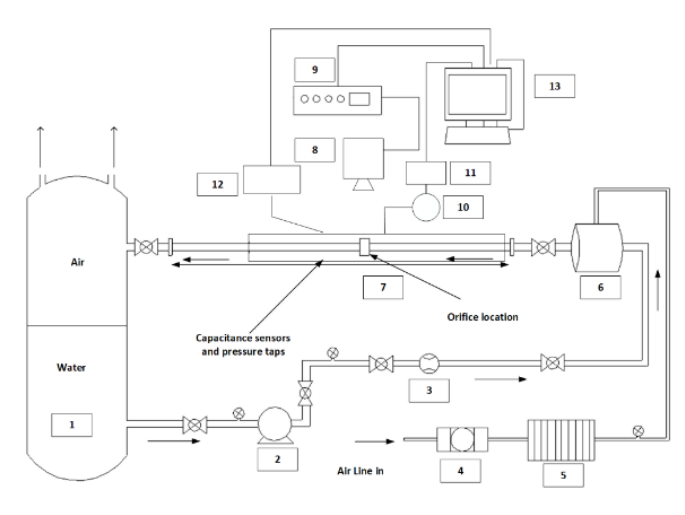

a) Schematic

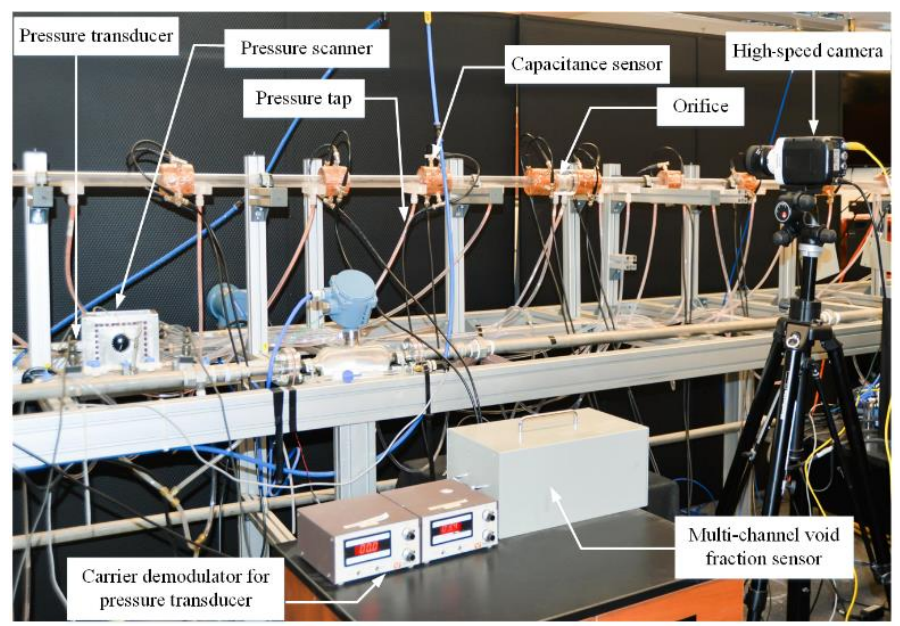

b) Actual image

Fig. 1: Experimental set-up.

from Validyne with an accuracy of $\pm 0.25 \%$ full-scale with a range of $350 \mathrm{kPa}$ and $35 \mathrm{kPa}$. Thus, the pressure at close locations can be measured by the $35 \mathrm{kPa}$ differential pressure transmitter with approximately $\pm 0.1 \mathrm{kPa}$ of error. The reference pressure in the test section was measured by a pressure transducer from Omega (model: PX481A), with an accuracy of $\pm 0.3 \%$ fullscale and a range of $414 \mathrm{kPa}$.

The flow pattern images were captured using a high-speed camera from Speedsense $\odot$ with a maximum resolution of $1920 \times 1200$ pixels and a trigger rate of up to 3000 frames per second. The test section was visualized at a distance of $13 \mathrm{~cm}$ upstream and $23 \mathrm{~cm}$ downstream of the restriction. The sampling rate of the recorded images was selected carefully based on the flow condition being considered.

The orifice was installed $3 \mathrm{~m}$ from the two-phase mixer. The mixture was transported through a horizontal straight pipe with an inner diameter of $25.4 \mathrm{~mm}$. The orifices had diameters of 6.35, 9.5, 12.7, and $19.05 \mathrm{~mm}$ that corresponded to the orifice-pipe area ratios of $0.062,0.14,0.25$, and 56 , respectively. The orifices were made of a clear acrylic material to allow for a visual inspection. The location of the orifice was selected based on the void fraction measurements for a straight pipe without restrictions. The void fraction measurements for a straight pipe without restrictions showed that the flow was fully developed at 70D from the two-phase flow mixer. Similarly, Salcudean et al. [13] reported a distance of 60D with the presence of restrictions and the same pipe diameter.

The void fraction was measured using a concave capacitance sensor as recommended by Ahmed [14]. This sensor consists of four electrodes made of copper attached to two half-hollow cylinders made of acrylic. The two half-hollow cylinders were clamped on the clear pipe at the point where the measurements of the void fraction were needed. Four plastic screws were used to tighten each capacitance sensor so it remained rigid during the experiment. The whole unit was shielded with a copper sheet to minimize any stray capacitance. A schematic of the concave capacitance sensor is presented in Fig. 1. The electrodes were connected through coaxial cables to an acquisition system built in the lab. The acquisition system was designed based on an LC circuit consisting of a parallel LC circuit, a frequency-to-digital converter FDC (TI FDC 2214 EVM evaluation by Texas Instruments), and an Arduino Due microcontroller. The main purpose of the design was to have an excitation frequency that was high enough for the sensor to overcome the conductivity of the measured medium and to have a high sampling frequency. In this design, the excitation frequency was $3.89 \mathrm{MHz}$ and the sampling frequency was 2.7 $\mathrm{kHz}$. Details of the void fraction acquisition system principles adopted in this work were presented by Elsaftawy et al. [15]. The void fraction was measured at twelve locations distributed equally upstream and downstream of the orifice. Twelve capacitance sensors and twelve acquisition circuits were built and connected to a personal computer through a USB cable to record the measurements. A LabVIEW@ interface was built to record and save the acquired measurements for further analysis. Repeatability tests for the void fraction measurements indicated that the measured data was within $\pm 2-5 \%$. Details of the pressure taps, and the void fraction sensor measurement locations are also shown in Table 1. 
Table 1: Locations of pressure taps and capacitance sensors along the test section.

Location from the orifice

Upstream

Downstream
Pressure taps, Z/D 94, 82, 70, 58, 46, 34, 22, 10, 0.5

$0.5,5,10,15,20,23,28,36,44,56,68,80,92$
Capacitance sensor, Z/D

$91.5,67,47.5,30,17.5,3.5$

$3,17.5,30.5,46.5,68.5,90.5$

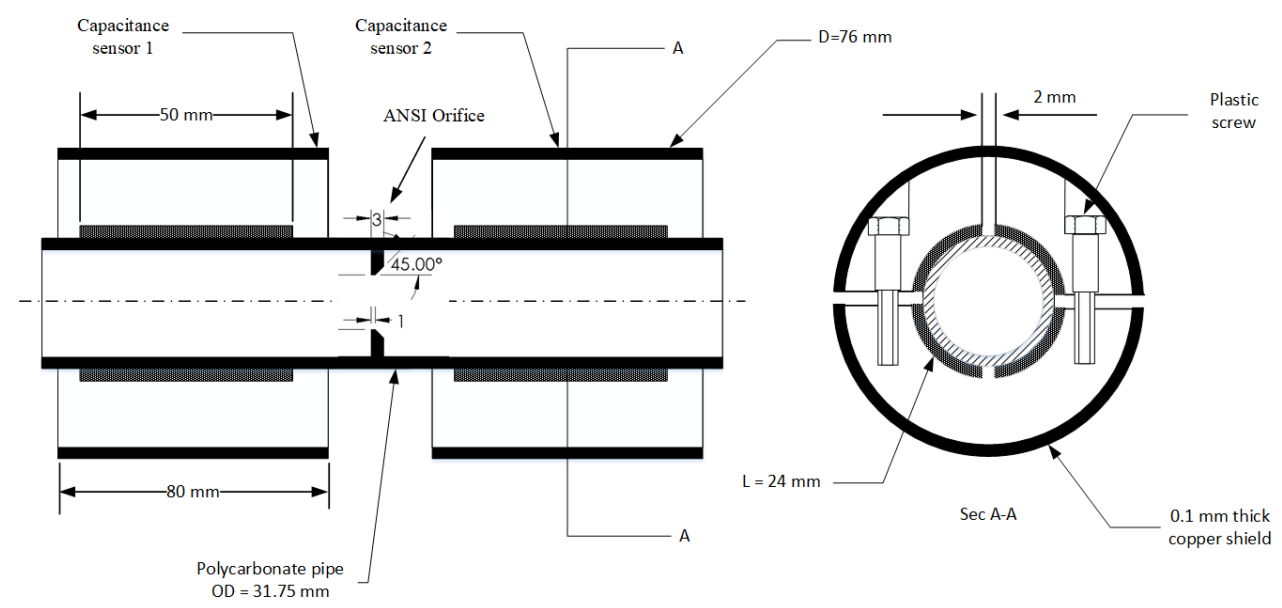

Fig. 1: Orifice and capacitance sensor dimensions used in this investigation.

\section{Results and Discussion}

The cases considered in this study were predicted by a Taitel and Duckler [3] flow pattern map, as shown in Fig. 2. It can be seen that all cases considered were within the intermittent flow pattern regime based on the upstream horizontal pipe. The effect of the area ratio on the flow pattern downstream of the orifice is discussed in the following sections.

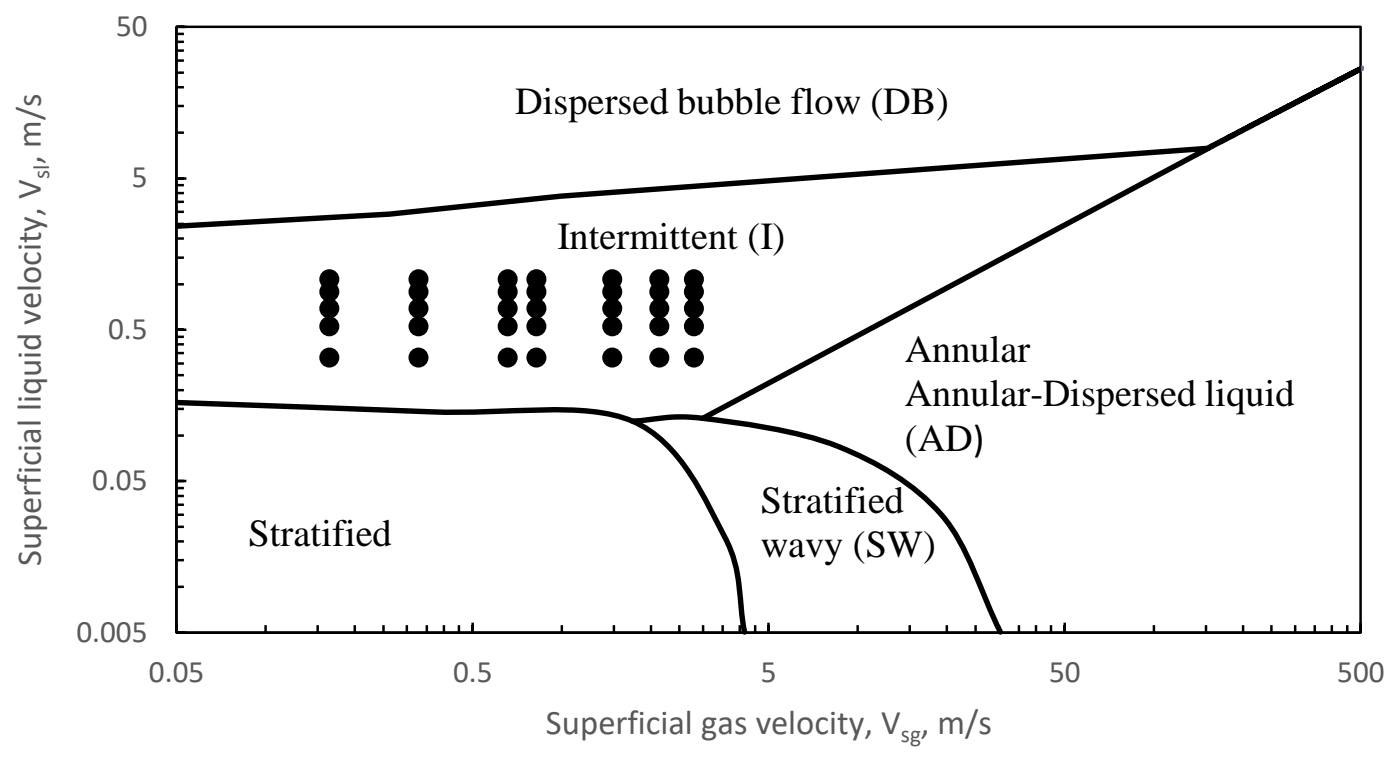

Fig. 2: Flow conditions considered in this study as predicted by a Taitel and Dukler [3] flow pattern map.

\subsection{Flow patterns observed downstream of the orifice}

Based on the experiments, a total of 86 void fraction data points were collected upstream and downstream of the orifice as well as 35 cases for the straight pipe without restriction. The void fraction was measured for a total time of $120 \mathrm{sec}$ and a sampling frequency of $2.7 \mathrm{kHz}$ to ensure a time-independent signal. The void fraction signal was used to acquire the PDF of the data. Since the flow pattern downstream of the orifice is different than the well-known flow 
patterns in the straight pipe case, the flow was visualized using a high-speed camera to understand the PDF of the void fraction signal. Six flow patterns were identified within a distance of 5D downstream of the orifice as follows:

1. Dispersed bubbles (DB): This flow pattern occurred at a low gas superficial velocity and a high liquid superficial velocity, especially at small area ratios. The dispersed bubbles formed when the elongated bubble broke up as it passed through the orifice. The dispersed bubbles occurred with a high turbulence. An example of the PDF and the corresponding flow can be seen in Fig. 3.
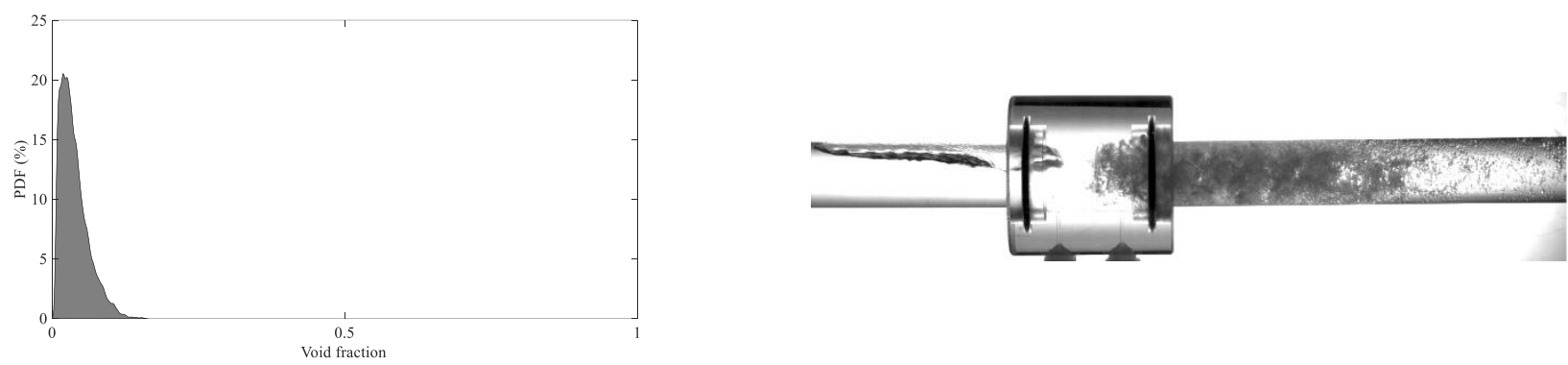
a) PDF
b) Image

Fig. 3: Dispersed bubble flow pattern downstream of the orifice.

2. Dispersed bubbles-liquid jet (DB-LJ): This transitional flow pattern occurred at a moderate gas superficial velocity. It consisted of the alternation of two flow patterns: dispersed bubbles and a liquid jet. Fig. 4 shows an example of the dispersed bubbles-liquid jet flow pattern.

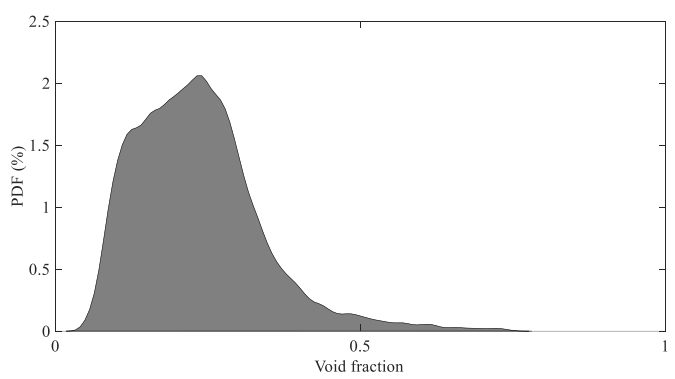

a) PDF

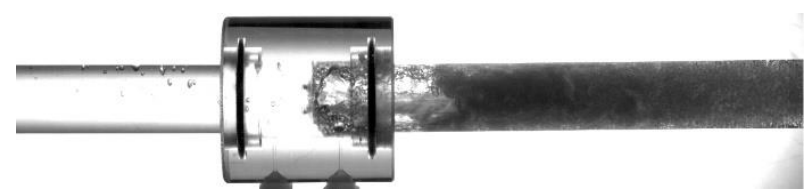

b) Image

Fig. 4: Dispersed bubbles-liquid jet flow pattern downstream of the orifice.

3. Dispersed bubbles-annular (DB-A): This flow pattern occurred at a large area ratio and a high liquid superficial $\mathrm{x}$ when

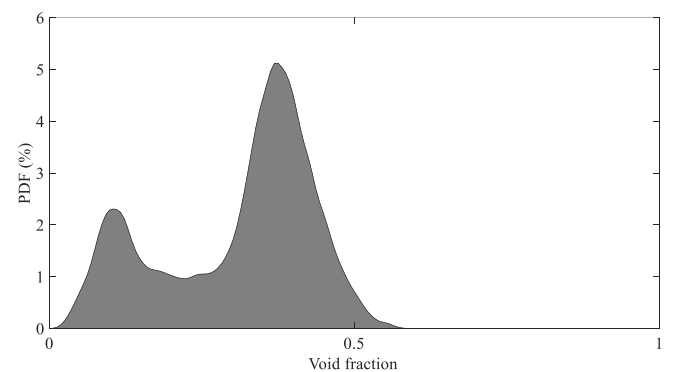

a) PDF

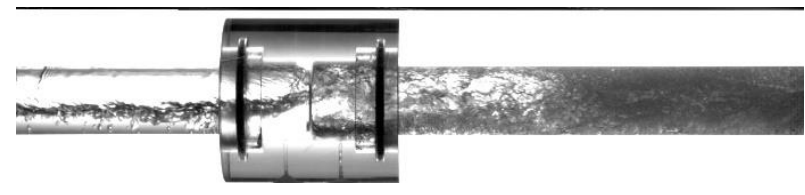

b) Image

Fig. 5: Dispersed bubbles-annular flow pattern.

the elongated bubble passed the orifice. An example of this flow pattern can be seen in Fig. 5.

4. Dispersed bubbles-slug (DB-S): This type of the flow pattern is a transition from a slug to dispersed bubbles and occurred at a large area ratio. An example of this flow pattern and its PDF can be seen in Fig. 6 . 

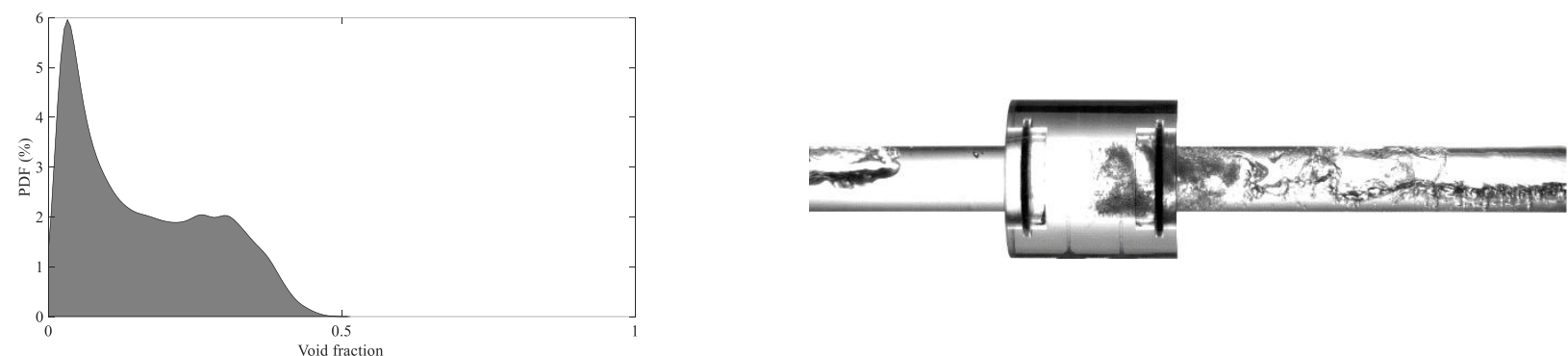

a) PDF

Fig. 6: Dispersed bubbles-slug flow pattern.

5. Liquid jet-annular (LJ-A): This flow pattern occurred at a small area ratio and a high gas superficial velocity. It consisted of the liquid jet formed when the liquid slug was passing through the orifice. The annular pattern was formed when the elongated bubble started to pass through the orifice. An example of this flow pattern is shown in Fig. 7.
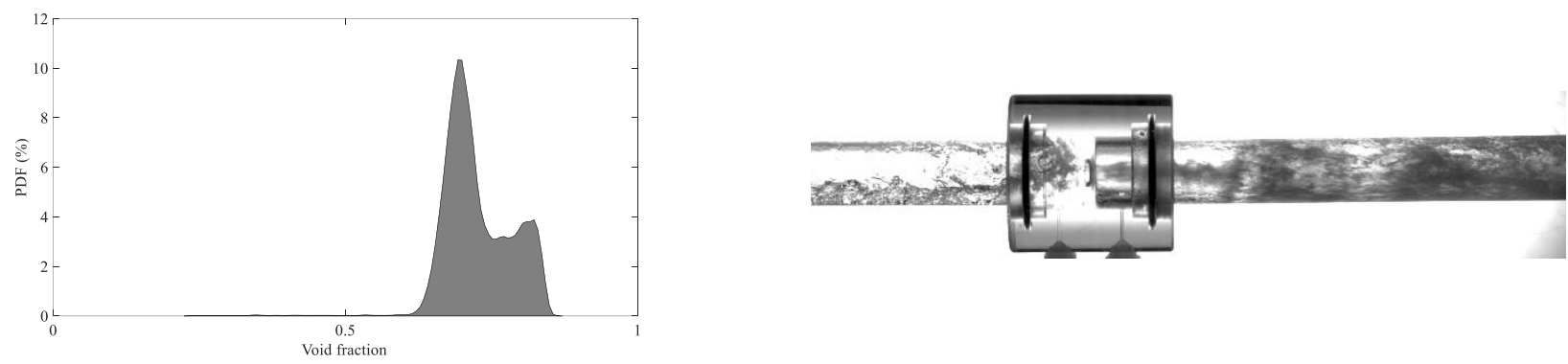

a) PDF

Fig. 7: Liquid jet-annular flow pattern.

6. Dispersed bubbles-liquid jet-annular (DB-LJ-A): This flow pattern is a transition between a dispersed bubble and a liquid jet-annular flow, in which both flow patterns alternate downstream of the orifice. An example of this flow pattern can be seen in Fig. 8.
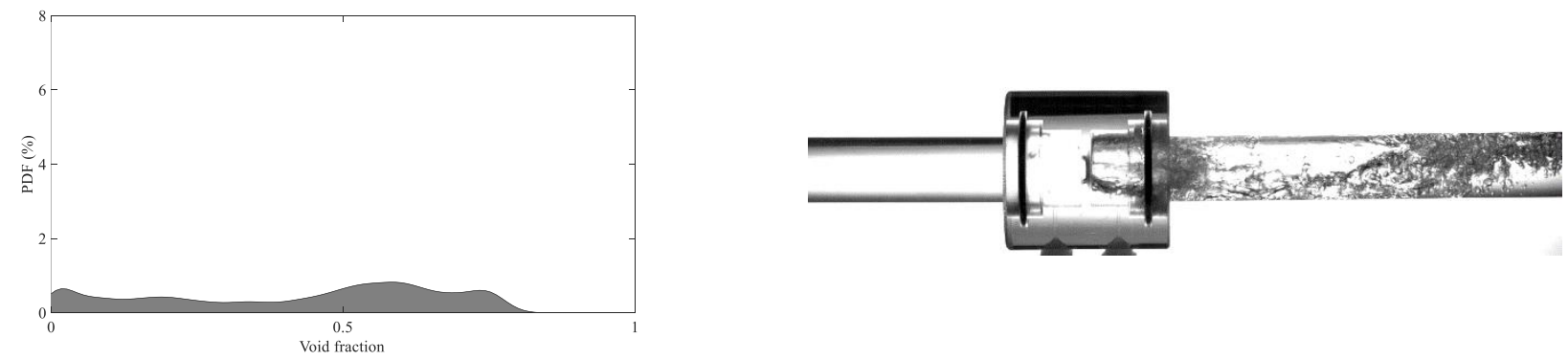

a) PDF

b) Image

Fig. 8: Dispersed bubbles-liquid jet-annular flow pattern.

Based on the classifications above, all 86 observations were identified, and the results are shown in Fig. 9. It should be noted that a slug flow pattern was observed in the straight pipe without restriction. It was found that the flow pattern cases could be appropriately represented by a total superficial velocity based on the orifice diameter versus the volumetric quality, as can be seen in Fig. 10. 


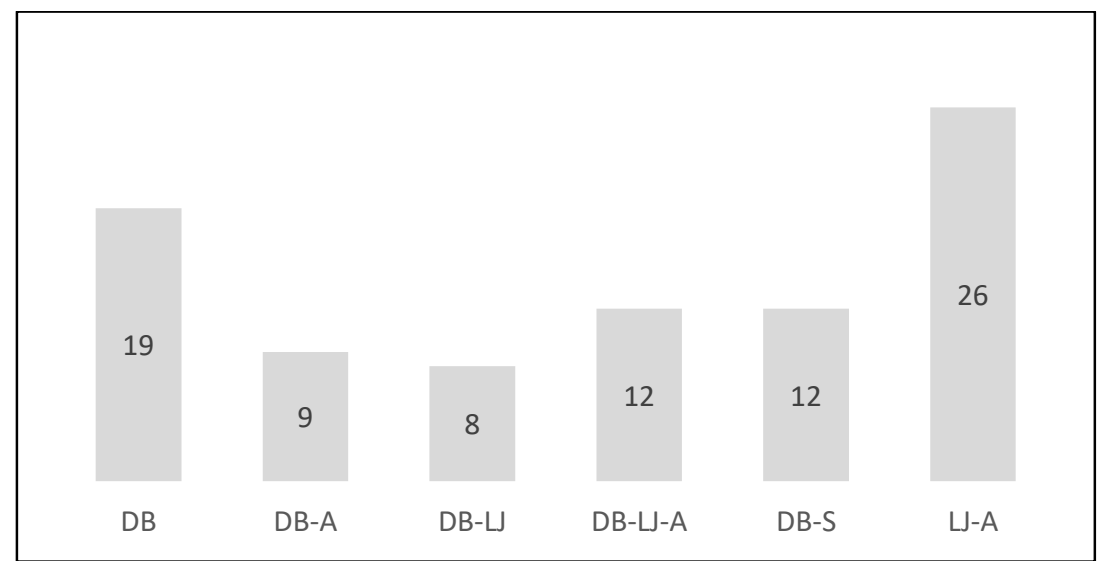

Fig. 9: Classification of the flow pattern observations based on the PDF of the void fraction data downstream of the orifice and the high-speed images.

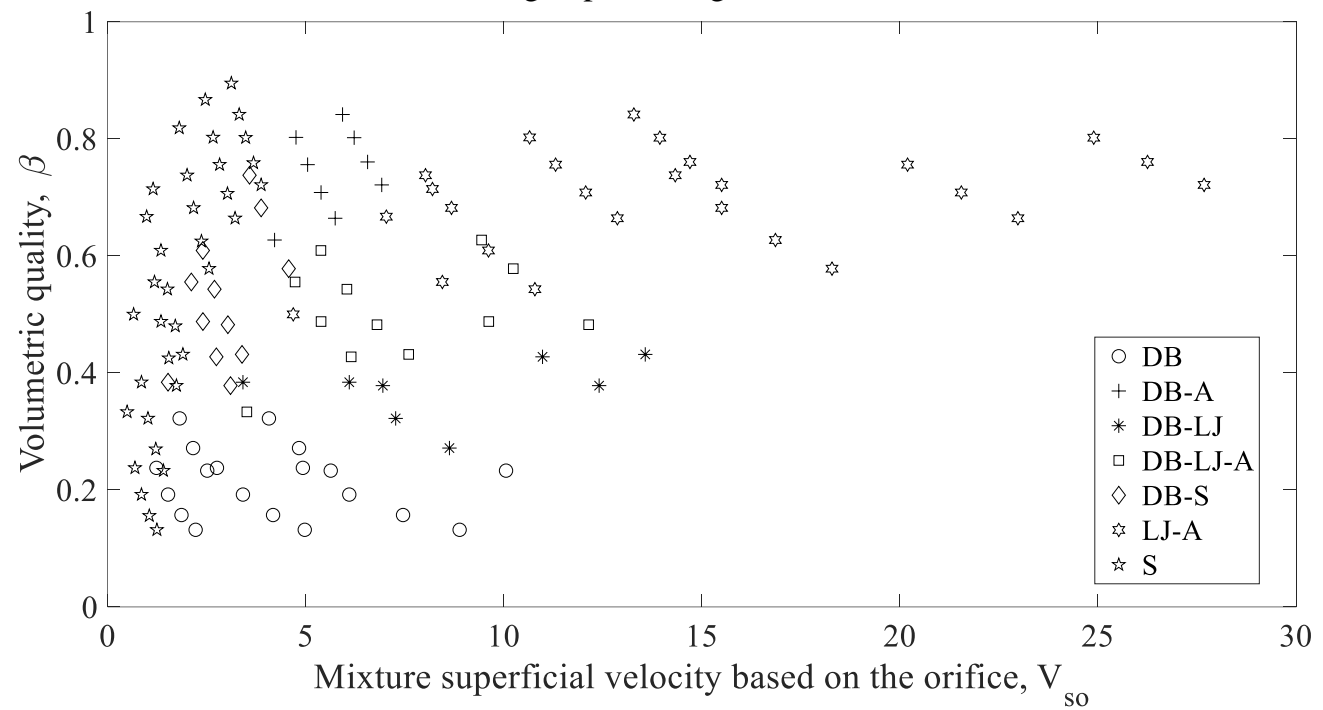

Fig. 10: Flow patterns identified based on the volumetric quality and the mixture superficial velocity based on the area of the orifice.

\subsection{Prediction of the flow pattern downstream of the orifice using Classification Learner}

For this method, the data was analyzed using the Classification Learner tool in MATLAB. In this tool, one can run an automated search for the best model by performing supervised machine learning using different models, as shown in Fig. 11. Supervised machine learning is done by defining the response to the input variables from a known source, such as experiments; thus, a database consisting of 86 observations with 12 predictors and one response were supplied to the Classification Learner tool. These predictors consisted of three independent variables: the area ratio, the liquid superficial velocity, and the gas superficial velocity. The rest of the variables were dependent ones: the local pressure drop; the liquid, the gas, and the mixture superficial velocity based on the area of the orifice; the mixture superficial velocity based on the area of the pipe; the averaged void fraction upstream and downstream of the orifice, and the ratio of both; and the volumetric quality. The response variable was the flow pattern downstream of the orifice that was acquired from the PDF and the highspeed images discussed in the previous section. A k-fold cross validation was used, in which the database was split into a number of folds. These folds were then trained, and the average model was obtained. For this analysis, it was found that $\mathrm{k}=5$ was the most appropriate number of folds to reduce the variance in the final model. The objective of this analysis was to find the model with the lowest error and the minimum number of predicters needed to predict the flow pattern downstream 


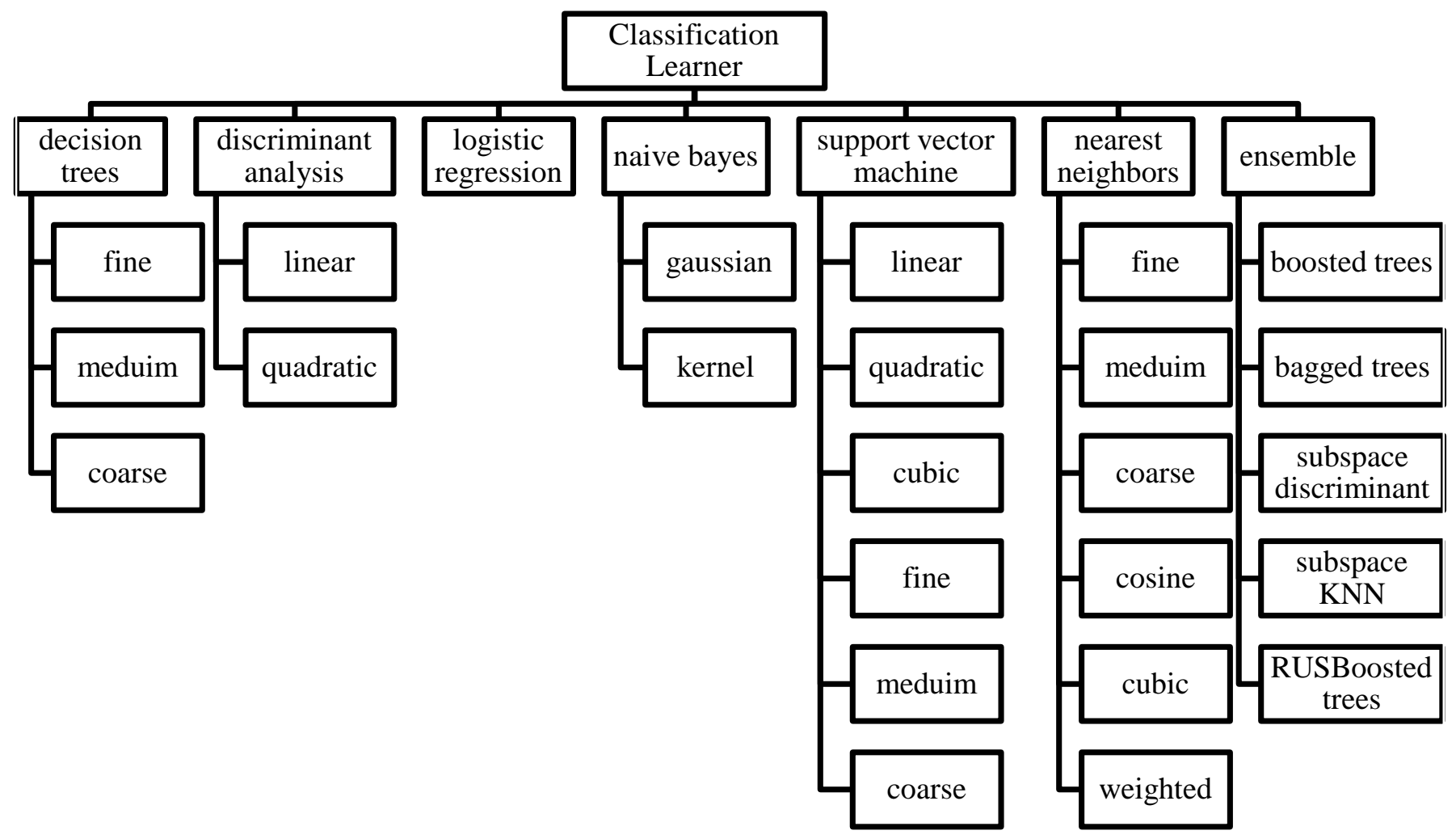

Fig. 11: Machine learning algorithms implemented in Classification Learner in MATLAB.

of the orifice; therefore, all twelve predictors and combinations made from them were used to achieve this goal. It was found that two predictors, namely the area ratio and the volumetric quality, which depends on the liquid and the gas superficial velocity, showed the lowest error at $9 \%$. The confusion matrix of this model was based on a weighted k-nearest neighbour or KNN algorithm and is presented in Table 2. A KNN algorithm is a simple and basic machine learning method that assumes that similar observations are close to each other in the future space.

Table 2: Confusion matrix of the flow pattern model prediction based on a weighted KNN algorithm.

\begin{tabular}{|c|c|l|l|l|l|l|l|}
\hline \multirow{2}{*}{} & \multirow{2}{*}{ Flow patterns } & \multicolumn{7}{|c|}{ Predicted class } \\
\cline { 2 - 8 } & \multicolumn{2}{|c|}{ DB } & DB-A & DB-LJ & DB-LJ-A & DB-S & LJ-A \\
\cline { 2 - 8 } & DB & $95 \%$ & 0 & $5 \%$ & 0 & 0 & 0 \\
\cline { 2 - 9 } & DB-A & 0 & $89 \%$ & 0 & $11 \%$ & 0 & 0 \\
\cline { 2 - 9 } & DB-LJ & $13 \%$ & 0 & $75 \%$ & $12 \%$ & 0 & 0 \\
\cline { 2 - 9 } & DB-LJ-A & 0 & 0 & $8 \%$ & $92 \%$ & 0 & 0 \\
\hline \multirow{2}{*}{} & DB-S & 0 & $17 \%$ & 0 & 0 & $83 \%$ & 0 \\
\hline & LJ-A & 0 & 0 & 0 & $4 \%$ & 0 & $96 \%$ \\
\hline
\end{tabular}

\section{Conclusions}

An investigation was performed on the flow pattern downstream of an orifice using machine learning algorithms. The flow pattern downstream of the orifice was identified using the PDF of the void fraction and a high-speed camera. 
A total of 86 experimental observations were classified into dispersed bubbles, dispersed bubbles-annular, dispersed bubblesliquid jet, dispersed bubbles-liquid jet-annular, dispersed bubbles-slug, and liquid jet-annular downstream of the orifice. The flow pattern downstream of the orifice was then modeled using the Classification Learner in MATLAB, which is based on supervised machine learning algorithms. It was found that the flow pattern can be predicted within an error of $9 \%$ by supplying only the volumetric quality and the area ratio. Predicting the flow pattern through the use of machine learning shows promise as a tool, and could be developed to cover more experimental data by expanding the database.

\section{Acknowledgements}

The authors would like to acknowledge the support received from the Natural Sciences and Engineering Research Council of Canada (NSERC) to carry out the present research. The authors would also like to acknowledge FloNergia Inc. for providing the materials and the expertise necessary to develop the measuring equipment.

\section{References}

[1] O. C. Jones, and J. N. Zuber, "The interrelation between void fraction fluctuations and flow patterns in two-phase flow," International Journal of Multiphase Flow, vol. 2, no. 3, pp. 273-306, 1975.

[2] J. M. Mandhane, G.A.Gregory, and K.Aziz, "A flow pattern map for gas-lquid flow in horizontal pipes," International Journal of Multiphase Flow, vol. 1, no. 4, pp. 537-553, 1974.

[3] Y. Taitel, and A. E. Dukler, "A model for predicting flow regime transitions in horizontal and near horizontal gasliquid Flow," The American Institute of Chemical Engineers Journal, vol. 22, pp. 47-55, 1976.

[4] Y. Mi, M. Ishii, and L. H. Tsoukalas, "Flow regime identification methodology with neural networks and two-phase flow models," Nuclear Engineering and Design, vol. 204, pp. 87-100, 2001.

[5] T. B. Trafalis, O. Oladunni, and D. V. Papavassiliou, "Two-phase flow regime identification with a multiclassification support vector machine (SVM) model," Industrial \& Engineering Chemistry Research Journal, vol. 44, no. 12, pp. 4414-4426, 2005.

[6] H. X. Wang, and F. Zhang, "Identification of two-phase flow regimes based on support vector machine and electrical capacitance tomography," Measurement Science and Technology, vol. 20, no. 11, pp. 1-8, 2009.

[7] H. Shaban, and S. Tavoularis, "Identification of flow regime in vertical upward air-water pipe flow using differential pressure signals and elastic maps," International Journal of Multiphase Flow, vol. 61, pp. 62 - 72, 2014.

[8] M. Al-Naser, M. Elshafei, and A. Al-Sarkhi, "Artificial neural network application for multiphase flow patterns detection: A new approach," Journal of Petroleum Science and Engineering, vol. 145, pp. 548-564, 2016.

[9] M. Ezzatabadipour, P. Singh, M. D. Robinson, P. Guillen-Rondon, and C. Torres, "Deep learning as a tool to predict flow patterns in two-phase flow," Proceeding in the Workshop on Data Mining for Oil and Gas, Houston, Texas, USA, 2017.

[10] W. Ahmed, "Evaluation of the proximity effect on flow-accelerated corrosion," Annals of Nuclear Energy, vol. 37, no. 4, p.p 598-605, 2010.

[11] W. H. Ahmed, M. M. Bello, M. El Nakla, A. Al Sarkhi, and H. M. Badr, "Experimental investigation of flow accelerated corrosion under two-phase flow conditions," Nuclear Engineering and Design, vol. 267, pp. 34-43, 2014.

[12] O. E. Bamidele, W. H. Ahmed, and M. Hassan, "Two-phase flow induced vibration of piping structure with flow restricting orifices," International Journal of Multiphase Flow, vol. 113, pp. 59-70, 2019.

[13] M. Salcudean, D. C. Groeneveld, and L. Leung, "Effect of flow-obstruction geometry on pressure drops in horizontal air-water flow," International Journal of Multiphase Flow, vol. 9, no. 1, pp. 73-85, 1983.

[14] W. Ahmed, "Capacitance sensors for void-fraction measurements and flow-pattern identification in air-oil two-phase flow," IEEE Sensors Journal, vol. 6, no. 5, pp. 1153-1163, 2006.

[15] A. Elsaftawy, J. Potts, and W. Ahmed, "Oscillation frequency LC-based sensor for characterizing two-phase flows in energy systems,” IEEE Sensors Journal, vol. 19, no. 1, pp. 65-77, 2019. 